Recepción: 05/10/2018

Evaluación: 05/11/2018

Aprobación: 06/05/2019

Artículo de Investigación-Científica

DOI: https://doi.org/10.22267/rhec.192222.56

\title{
Comunidad virtual de práctica para desarrollo de competencias en la construcción de software en equipo ${ }^{1}$
}

\author{
Álvaro Alexander Martínez Navarro ${ }^{2}$ \\ Universidad Mariana, Colombia \\ Franklin Eduardo Jiménez Giraldo ${ }^{3}$ \\ Universidad Mariana, Colombia \\ Giovanni Albeiro Hernández Pantoja ${ }^{4}$ \\ Universidad Mariana, Colombia \\ Robinson Andrés Jiménez Toledo ${ }^{5}$ \\ Universidad Mariana, Colombia
}

\section{Resumen}

Este artículo es resultado de la investigación: Incidencia de las Comunidades Virtuales de Práctica en el desarrollo de competencias para la construcción de software en los programas de Ingeniería de Sistemas del Sur-Occidente Colombiano. El objetivo principal de esta investigación fue aportar al desarrollo

$1 \quad$ Este artículo es resultado de la investigación: Incidencia de las Comunidades Virtuales de Práctica en el desarrollo de competencias para la construcción de software en los programas de Ingeniería de Sistemas del Sur-Occidente Colombiano, avalada por la Universidad Mariana, San Juan de Pasto, Colombia.

2 Ingeniero de Sistemas. Magíster en Docencia Universitaria. Master en Análisis y Visualización de Datos Masivos. Docente de la Universidad Mariana. Grupo de investigación: GISMAR. Línea de Investigación: Didáctica de la Ingeniería. Correo electrónico: amartinez@, umariana.edu.co. Código ORCID: https://orcid.org/0000-0002-6341-4863

3 Ingeniero de Sistemas, Magíster en Software Libre. Docente de la Universidad Mariana. Grupo de investigación: GISMAR. Línea de Investigación: Didáctica de la Ingeniería. Correo electrónico: fjimenez@umariana.edu.co. Código ORCID: https://orcid.org/0000-0002-3071-7198

4 Ingeniero de Sistemas. Magíster en Docencia Universitaria. Docente de la Universidad Mariana. Grupo de investigación: GISMAR. Línea de Investigación: Didáctica de la Ingeniería. Correo electrónico: gihernandez@umariana.edu.co. Código ORCID: https:// orcid.org/0000-0001-7903-6513

5 Ingeniero de Sistemas. Magíster en Docencia Universitaria. Docente de la Universidad Mariana. Grupo de investigación: GISMAR. Línea de Investigación: Didáctica de la Ingeniería. Correo electrónico: rjimenez@umariana.edu.co. Código ORCID: https://orcid. org/0000-0003-2218-0850 
de competencias para la construcción de software basada en equipo, en los estudiantes de Ingeniería de Sistemas de la Universidad Mariana, mediante el uso de una Comunidad Virtual de Práctica (CVP). Este trabajo, se desarrolló bajo el paradigma cuantitativo, con un enfoque empírico-analítico de tipo descriptivo y propositivo. La población objeto de estudio corresponde a los estudiantes de séptimo y octavo semestre. Como resultado, se identifica el nivel de desempeño de los estudiantes en las dimensiones cognitiva (Saber) y aptitudinal (Saber-hacer) antes de formar parte de la CVP. Se diseña y pone en funcionamiento una CVP, fundamentada en el aprendizaje basado en problemas y estructurada mediante: identidad, práctica y roles que deben cumplir los integrantes; para propiciar en los miembros que la conforman, el desarrollo de competencias para la construcción de software basada en equipo, de manera complementaria al trabajo que se realiza en la Universidad. La investigación permite concluir que la CVP, impacta de manera positiva y hace posible avanzar, a los integrantes que se desempeñaron como miembros activos de la comunidad, en los niveles de logro alcanzados para las dimensiones: cognitiva y aptitudinal; consiguiendo aumentar la posibilidad de vinculación en un mercado laboral para construir software que está en constante crecimiento.

Palabras clave: Competencia profesional; Comunidad Virtual de Práctica; Construcción de software en equipo; Informática educativa. 


\title{
Practicum virtual community to develop competencies in the building of team-made software
}

\begin{abstract}
This research paper aims to analyze the incidence of the Practicum Virtual Communities in the development of competencies when building software in the Systems Engineering programs in southwestern Colombia, specifically with students from Mariana University through the use of a Practicum Virtual Community (PVC). This work was developed under the quantitative paradigm, with a descriptive and propositive empirical-analytical approach. The targeted population was students from seventh and eighth semester. As a result, the level of performance of the students is identified in the cognitive (know) and aptitudinal (know-how) dimensions before being part of the PVC. Then, a PVC was designed and implemented, using problem-based learning and following this structure: identity, practice and roles to be fulfilled by the community members, to foster the development of competencies in the building of team-made software. As a conclusion, the impact of the PVC was vastly positive within the community members both in the cognitive and aptitude level. In the end, these members proved their abilities when building software and were the most suitable to compete for a job position.
\end{abstract}

Keywords: Professional competencies; Practicum Virtual Community; teammade software; educational engineering. 


\section{Comunidade virtual de prática para desenvolvimento de habilidades na formação de software en equipes}

\section{Resumo}

Este artigo é o resultado da pesquisa: Impacto das comunidades virtuais de prática no desenvolvimento de competências para a construção de software nos programas de engenharia de sistemas do sudoeste da Colômbia. O principal objetivo desta pesquisa foi contribuir para o desenvolvimento de habilidades para a construção de software em equipe, nos estudantes de Engenharia de Sistemas da Universidade Mariana, através do uso de uma Comunidade Virtual de Prática (CVP). Este trabalho foi desenvolvido sob o paradigma quantitativo, com abordagem empírico-analítica, do tipo descritivo e propositivo. A população estudada corresponde a alunos do sétimo e oitavo semestre. Como resultado, é identificado o nível de desempenho do aluno nas dimensões cognitiva (Conhecimento) e aptidão (saber-fazer) antes de fazer parte do CVP. Um CVP é projetado e colocado em operação, com base na aprendizagem baseada em problemas e estruturado por meio de: identidade, prática e funções que os membros devem cumprir; promover nos integrantes que o compõem, o desenvolvimento de habilidades para a construção de software em equipe, de forma complementar ao trabalho realizado na Universidade. A pesquisa permite concluir que o CVP afeta positivamente e possibilita avançar, Aos integrantes que trabalhavam como membros ativos da comunidade, nos níveis de realização alcançados para as dimensões: cognitiva e de capacidades; procurando aumentar a possibilidade de ligação em um mercado de trabalho para criar software que está em constante crescimento.

Palavras-chave: competência profissional; Comunidade virtual de prática; construção de software em equipe; Informática educacional. 


\section{Introducción}

Las tecnologías de la información y las comunicaciones (TIC) se han consolidado como una herramienta propicia para potencializar y transformar la educación, en especial la Educación Superior. ${ }^{6}$ En este sentido, las Instituciones de Educación Superior (IES), como responsables del fomento del conocimiento en todos los ámbitos de la sociedad, ven en las TIC a un valioso aliado. ${ }^{7}$ El uso de las TIC en estos procesos conlleva modificar enfoques pasivos y tradicionales de la Educación Superior para enfrentar el desafío que impone una sociedad donde abunda la información. ${ }^{8}$ En este contexto, las comunidades virtuales de práctica (CVP) aparecen como un aporte importante a la innovación académica, al introducir un cambio, tanto en el ámbito de la modalidad (de presencial a virtual), como en la conceptualización de los procesos de formación de los estudiantes, basado en una cultura de aprendizaje autónomo, colaborativo y de conocimiento compartido, fundamentado en el desarrollo de competencias por medio de la práctica. ${ }^{9}$

Según Rheingold,${ }^{10}$ una comunidad virtual es la sinergia social que surge a partir del uso de los recursos que proveen las TIC, cuando, en la interacción de las personas, se generan reflexiones en la búsqueda de sentido y significado para los integrantes. De acuerdo con Salinas, ${ }^{11}$ existen diferentes tipos de comunidades virtuales. Un tipo de comunidad virtual es la de aprendizaje, ${ }^{12}$ en que los estudiantes comparten intereses comunes, y las TIC sirven como medio para que los estudiantes interactúen, para desarrollar actividades y compartir información. Este tipo de comunidad virtual se centra en la reflexión sobre ¿qué? y ¿cómo?

6 Rama Kummitha y Satyajit Majumdar, "Dynamic curriculum development on social entrepreneurship - A case study of TISS", The International Journal of Management Education, vol. 13. No. 3, (2015): 260-267.

7 Daniela Dumitru y Vladimir Enăchescu, "Communities of Practice as a mean for decentralization”, Procedia - Social and Behavioral Sciences. No. 187 (2015): 752-756.

8 Daciana Lupu y Andreea Laurenţiu, "Using New Communication and Information Technologies in Preschool Education”, Procedia - Social and Behavioral Sciences. No. 187 (2015): 206-210.

$9 \quad$ Hyung Jin Kim et al., "Third-party mobile app developers' continued participation in platform-centric ecosystems: An empirical investigation of two different mechanisms", International Journal of Information Management, vol. 36. No 1 (2016): 44-59.

10 Howard Rheingold, La comunidad virtual: una sociedad sin fronteras (Barcelona: Gedisa, 1996).

11 Jesús Salinas, “Comunidades virtuales y aprendizaje digital”, Edutec'03 (2003): 1-21.

12 Ibíd. 
construir conocimiento. Una comunidad virtual de práctica ${ }^{13}$ coincide en buscar el aprendizaje como un fin de la sinergia grupal; sin embargo, Salinas plantea que va más allá y se centra en que el aprender es un acto natural que debe ser similar a la cotidianidad de un entorno no escolarizado; es decir, en este tipo de comunidad se generan roles (no únicamente existen el docente y el estudiante) para apoyar al grupo y participar de manera activa.

Una comunidad virtual de práctica puede definirse como un grupo de personas que comparte un interés mutuo en un dominio específico, para lo cual participan en un proceso de aprendizaje colectivo. ${ }^{14}$ En una comunidad virtual de práctica, los miembros (usuarios) son personas con ideas afines, pero geográficamente dispersos. Estos miembros no son pasivos en la construcción de saberes en línea, sino, también, los crean y comparten, para desarrollar conocimientos, habilidades y actitudes sobre un tema específico de su interés. ${ }^{15}$ Estas comunidades virtuales de práctica tienen múltiples niveles y tipos de participación: los miembros pueden ser participantes centrales en una comunidad y, al mismo tiempo, ser participantes periféricos en otras. Incluso dentro de una misma comunidad, los miembros pueden ir y venir entre el núcleo y la periferia. ${ }^{16}$

Por otra parte, diversos factores han revolucionado las formas cómo los estudiantes, también, interactúan con el mundo. Hoy en día, es mucho más fácil acceder a la información y existe una amplia difusión de información en Internet, redes sociales y dispositivos móviles. Estos elementos los utilizan de forma cotidiana los estudiantes, por lo que llevar la educación a estos lugares u objetos es una prioridad, que debe aportar para cubrir las exigencias de un mercado globalizado, que cada día exige mayor movilidad y agilidad en la información. ${ }^{17}$

13 Ibíd.

14 Yonggang Pan et al., "Integrating social networking support for dyadic knowledge exchange: a study in a virtual community of practice", Information \& Management, vol. 52. No. 1 (2015): 61-70.

15 Francesco Rogo et al., "Assessing the performance of open innovation practices: A case study of a community of innovation", Technology in Society. No. 38 (2014): 60-80.

16 Nicole V. S. Ratzinger-Sakel y Glen L. Gray, "Moving toward a learned profession and purposeful integration: Quantifying the gap between the academic and practice communities in auditing and identifying new research opportunities", Journal of Accounting Literature. No. 35 (2015): 77-103.

17 Maria-Iuliana Dascalu et al., "Improving e-learning communities through optimal composition of multidisciplinary learning groups", Computers in Human Behavior. No. 30 (2014): 362-371. 
La movilidad y agilidad de la información, aportada por el sector de las TIC, brinda oportunidades únicas para el aumento y evolución de la economía, la política, el sector social y cultural, a cualquier país. ${ }^{18}$ Por ejemplo, en países como la India, la industria de software genera fuentes de trabajo bien remuneradas. ${ }^{19}$ El sur-occidente de Colombia no ha sido ajeno a esta dinámica y viene avanzando de manera importante en esta "nueva economía", debido a que algunas firmas (empresas dedicadas a la construcción de software) ya se encuentran posicionadas y compiten por este mercado potencial. ${ }^{20}$

Esto abre oportunidades, como demanda de profesionales, en el desarrollo de software, con el salario más competitivo de América Latina, para lo cual se exige que los trabajadores fuesen competentes en la mayoría de aspectos incluidos en el desarrollo de software ${ }^{.21}$ Con base en las oportunidades laborales que presenta la industria del software, según el informe presentado por Infosys y Universidad EAFIT ${ }^{22}$ al Ministerio de Tecnologías de la Información y las Comunicaciones de Colombia, se prevé que, para el año 2020, harían falta 166.956 profesionales en la industria TI del país. Este reto obliga a las Instituciones de Educación Superior a plantear alternativas que permitieran el acceso a las personas a una formación que avance de consumidores a productores de software .23 $^{23}$

La construcción de software es una actividad compleja que, actualmente, se realiza por equipos, que deben dar respuesta a las necesidades crecientes de software en las organizaciones. No obstante, el trabajar en equipo presenta un conjunto de factores que inciden en el momento de alcanzar los objetivos que esta

18 Christopher J. Robertson et al., "An analysis of the predictors of software piracy within Latin America", Journal of Business Research, vol. 61. No. 6 (2008): 651-656.

19 Sriram Narayanan et al., "The antecedents of process integration in business process outsourcing and its effect on firm performance", Journal of Operations Management, vol. 29. No. 1 (2011): 3-16.

20 Giovanni Hernández et al., "Metodología adaptativa basada en Scrum: Caso empresas de la Industria de Software en San Juan de Pasto - Colombia". Revista Tecnológica-ESPOL, vol. 28. No. 5 (2015): 211-223.

21 Fedesoft. "Visión estratégica del sector de software y servicios asociados. Plan de mercadeo y ventas regionalizado del sector en Colombia". Bogotá: MINTIC y Colciencias, 2013.

22 Infosys y Universidad EAFIT. Brecha de Talento Digital Infosys - Universidad EAFIT (Medellín: Universidad EAFIT e Infosys Limited, 2014).

23 Giovanni Hernández et al., "Scrum y Peopleware: elementos clave para la gestión en la construcción de software.» Risti. No. E19 (2019): 265-277. 
labor traza. Putnam ${ }^{24}$ logra demostrar que, cuando el tamaño del equipo crece por encima de un grupo de personas, el esfuerzo se aumenta, pero el tiempo del proyecto no se reduce. DeMarco ${ }^{25}$ plantea que un equipo con exceso de trabajo, ocupado y sobresaturado, no es garantía de mayor efectividad y, además, no permite visualizar mayores beneficios para un proyecto. Así mismo, Brooks, ${ }^{26}$ a partir de la experiencia, éxito y fracaso en el desarrollo de software, logra concluir que, en un equipo de desarrollo, agregar más personas a un proyecto que tiene un retraso provocará un retraso mayor. En trabajos intelectuales, como programar, escribir artículos, entre otros, las interrupciones son un mal que afecta enormemente a la productividad. Parnin y Rugaber ${ }^{27}$ concluyen que, normalmente, a un programador le llevara de 10 a 15 minutos volver al estado de concentración previo al haber sido interrumpido. Otro aspecto fundamental, que incide en la productividad y desempeño de un equipo de trabajo, es la comunicación. Los estudios realizados por Cockburn ${ }^{28}$ permiten establecer que la forma más efectiva de comunicación corresponde a mantener al equipo interactuando en un sitio, frente a un tablero, formulando y respondiendo cuestionamientos e interrogantes. Este corresponde a un gran desafío en un periodo en que el teletrabajo y la contratación freelance se han convertido en una práctica habitual, por la escasez de mano de obra calificada para el desarrollo de software.

El término comunidad de práctica lo estudió y concibió Wenger, ${ }^{29}$ en su labor de observar y analizar el conocimiento que se difunde desde una comunidad científica y cuando buscaba potenciar este aspecto a nivel corporativo como una institucionalización de la antigua «tormenta o lluvia de ideas» o de intercambios informales.

24 Lawrence Putnam, "A general empirical solution to the macro software sizing and estimating problem”. IEEE transactions on Software Engineering, vol. SE-4. No. 4 (1978): $345-$ 361.

25 Tom DeMarco. Slack: Getting past burnout busywork and the myth of total efficiency (New York: Broadway Books, 2001).

26 Frederick Brooks. The Mythical Man-Month: Essays in Software Engineering (Boston: Addison-Wesley, 1995).

27 Chris Parnin y Spencer Rugaber, "Resumption strategies for interrupted programming". Software Quality Control, vol. 19, No. 1 (2011): 5-34.

28 Alistair Cockburn, Effectiveness of different modes of communication graph. (2015), http:// alistair.cockburn. us/Effectiveness + of + different + modes + of + communication + graph.gif

29 Etienne Wenger, “Communities of Practice: Learning as a Social System”, Systems thinker, vol. 9. No. 5 (1998): 1-10. 
La finalidad de una comunidad virtual de práctica es explicitar la transferencia informal de conocimiento, para ofrecer una estructura formal que permite adquirir más conocimiento a través de las experiencias compartidas dentro del grupo. Por esta razón, la propia identidad del grupo se refuerza al potenciar el aprendizaje como un proceso de participación y liderazgo compartido. Este tipo de comunidad no es científico, ya que su planteamiento no es la ciencia, sino la experiencia de la práctica y la gestión compartida del conocimiento. Esta gestión del conocimiento se realiza siempre de una forma colaborativa y en un proceso continuo de establecer estrategias de participación, liderazgo, identidad y aprovechamiento del conocimiento. ${ }^{30}$ Existen diferentes tipos de comunidades de práctica, entre los que se encuentran: pequeños o grandes grupos, de larga o corta duración, presencial o virtual, sincrónicos o asíncronos, homogéneos o heterogéneos; y espontáneos o intencionales.

Las comunidades virtuales de práctica se comprenden como entornos de aprendizaje basados en tecnología de la información y las comunicaciones, que agrupan personas relacionadas con una temática específica, que comparten documentos, recursos, ideas, pensamientos; es decir, explotan las posibilidades de las herramientas de comunicación en Internet para un beneficio en común.

En este artículo, una competencia se comprende como un conjunto de estructuras complejas en las dimensiones cognitiva (Conocimientos), aptitudinal (Habilidades) y afectivo-motivacional (Actitudes) ${ }^{31}$ que permite solucionar problemas propios de la construcción de software basada en equipos. En este sentido, la comunidad virtual de práctica pretende cubrir aspectos no incluidos dentro de la academia, donde, generalmente, se enseña a desarrollar software de manera individual, por imitación y utilización de ejercicios descontextualizados.

En la trayectoria del tema de trabajo, existen estudios, como los de Forero y Mesa, ${ }^{32}$ donde se desarrolla una reflexión sobre el proceso de evolución de la educación virtual en el ámbito de la Educación Superior, en Colombia. Este tra-

30 Zoia Bozu y Francesc Muñoz, "Creando comunidades de práctica y conocimiento en la Universidad: una experiencia de trabajo entre las universidades de lengua catalana". Universities and Knowledge Society Journal, vol. 6. No. 1 (2009): 1-10.

31 Tobón, Sergio, La formación basada en competencias en la educación superior: El enfoque complejo (México: Universidad Autónoma de Guadalajara, 2008).

32 Aracely Forero y Fredy Mesa, "Reflexiones para la historia y prospectiva de la virtualidad en la educación superior colombiana", Historia de la Educación Colombiana, vol. 15. No. 15 (2012): 215-236. 
bajo inicia con un análisis de dos estudios: el primero corresponde a la memoria de la investigación, en la década de los años 90, y el segundo es un diagnóstico de la Educación Superior virtual, realizado a finales de 2002. Posteriormente, se establece una reflexión sobre los programas que, en Colombia, han propiciado el desarrollo y avance de las TIC, y su aplicación educativa, hasta el año 2010. Por último, se adelanta un análisis de las tendencias en la educación virtual. Los principales resultados permiten intentificar que la virtualización de la Educación Superior en Colombia sigue creciendo y, desde el año 2010, recibe apoyo a través de políticas estatales, con el fin de mejorar indicadores de cobertura. Además, los autores recomiendan que se debe seguir avanzando en la incorporación de tecnologías de punta y emergentes en la educación.

Existen trabajos, como el de Pan y otros, ${ }^{33}$ en los que se propone una comunidad virtual de práctica, como un sistema independiente de gestión del conocimiento; para esto, integran redes sociales de apoyo en beneficio de la comunidad virtual de práctica; para compartir el conocimiento, utilizan foros de discusión. El estudio de campo, realizado en este trabajo, concluye que el apoyo de las redes sociales intensifica el intercambio de conocimientos por parejas de actores, con aclaración respecto a que el diálogo entre amigos incrementa la probabilidad de colaboración e intercambio de conocimientos.

Por otra parte, Rodríguez, Busco y Flores; ${ }^{34}$ y Rogo, Cricelli y Grimaldi ${ }^{35}$ señalan que las comunidades virtuales de práctica desempeñan un papel importante en los procesos de innovación, pero no dejan en claro cuál es su función particular. En este artículo, los autores buscan diferenciar los tipos de conocimientos adquiridos con la práctica, con el fin de aplicarlos en innovación empresarial de una manera colaborativa. Para lograrlo, plantean elementos deductivos, que destacan diferentes impulsos de reflexividad y diferentes lógicas internas de la reflexividad.

En relación con el aprendizaje, virtual Ratzinger-Sakel y Gray ${ }^{36}$ proponen propiciar las condiciones para que surgiera y se formara una comunidad virtual de práctica en el estudio de la contabilidad, para enfocarse sobre la profesión

\footnotetext{
33 Pan et al., ““'Integrating social networking...”, 61.

34 Darío Rodríguez et al., "Information technology within society's evolution", Technology in Society. No. 40 (2015): 64-72.

35 Rogo et al., "Assessing the performance of open...", 60.

36 Ratzinger-Sakel y Gray, "Moving toward a learned profession...", 77.
} 
de auditoría, para lo cual plantean construir un compendio de lo aprendido en la profesión, investigación contable, las prácticas estudiantil y profesional, de contabilidad. Para esto, se integran temas de interés para la comunidad virtual de práctica, con énfasis en la visión compartida de un cuerpo de conocimiento en temas contables de auditoría.

Jiménez-Zarco, González-González, Saigí-Rubió y Torrent-Sellens ${ }^{37}$ describen la manera como se propician condiciones para que se conformase una comunidad virtual de práctica en la apropiación de conceptos del sector sanitario, por lo que se propone un espacio de aprendizaje colaborativo, con utilización de una red social, donde se vinculan personas con diferentes rasgos, pero con un interés en común, para intercambiar información, experiencias y contenidos relacionados con el dominio del sector sanitario. La CVP tiene el propósito de crear, compartir conocimientos y aprender colaborativamente, para lograr beneficios relacionados con el diagnóstico y el tratamiento de los pacientes en relación con el costo, realizar una gestión más eficiente, incrementar la calidad y precisión del diagnóstico. Además, se pretendió aumentar el capital social de los participantes y crear redes de personas de confianza, dado el interés en este tema. Los resultados de participación en la comunidad virtual de práctica demuestran que una intervención activa y colaborativa impacta de manera positiva en cada uno de los miembros, con un incremento en el interés por el tema tratado.

En el trabajo realizado por Dascalu, Bodea, Lytras, De Pablos y Burla$\mathrm{cu},{ }^{38}$ se propone componer grupos de aprendizaje efectivos, donde los miembros tienen diferentes antecedentes de conocimiento en un dominio dado. El estudio define que la cuantificación de diversos indicadores, como el fondo de la diversidad y la similitud entre el tipo de interés de los participantes dentro de un grupo y entre los grupos, puede tener un impacto positivo en brindar condiciones para que una comunidad virtual de práctica surgiera y se formara, con un enfoque principalmente en el aprendizaje continuo de sus miembros.

\footnotetext{
37 Ana Isabel Jiménez-Zarco et al., "The co-learning process in healthcare professionals: Assessing user satisfaction in virtual communities of practice", Computers in Human Behavior, vol. 51. Parte B (2015): 1303-1313.

38 Dascalu et al., "Improving e-learning communities through...", 362.
} 
En los trabajos presentados por Ordóñez, Hernández, Ordóñez, Ordóñez y $\operatorname{Cobos}^{39}$ y Ordóñez, Ordóñez, Ordóñez, Cobos y Hernández ${ }^{40}$ se plantea potencializar las competencias para el desarrollo de software, en estudiantes de Informática y carreras afines. Esta investigación divide las competencias de los estudiantes, para esta área, en seis ejes y demuestran como, a través de la comunidad virtual de práctica, se logra avanzar en el desarrollo de competencias mediante la construcción de conocimiento de manera colaborativa y apoyado por casos de estudio.

En relación con los antecedentes revisados, la investigación que sustenta este artículo tiene como similitudes el uso de una comunidad virtual de práctica como medio para desarrollar competencias en un área específica de conocimiento, establece las competencias y niveles de aprendizaje que se desea alcanzar y se apoya en otros recursos computacionales, como las redes sociales y entornos virtuales de aprendizaje. No obstante, en este trabajo investigativo, se presenta como novedad, en primer lugar, clasificar las competencias en tres dimensiones (conocimientos, aptitudes y actitudes) y trabajar con las dimensiones: cognitiva y aptitudinal. Además, se elaboró una planeación, que parte de las competencias que se desean desarrollar, a partir de las cuales se definen los niveles de aprendizaje y se utiliza como estrategia los casos de estudio. Así mismo, se organizan los recursos y su forma de uso y se posibilita que la comunidad virtual de práctica se auto-organizará y auto-gestionará. De igual forma, aún no se ha planteado la formación de una comunidad virtual de práctica que permita a un conjunto de estudiantes de Ingeniería de Sistemas, ubicados geográficamente distantes, reunirse en un espacio virtual, donde puedan potencializar colaborativamente su conocimiento, habilidades y actitudes en la construcción de software basada en equipos desde un enfoque ágil, a través del intercambio de experiencias, buenas prácticas y conocimientos entre todos los miembros de la comunidad.

39 Camilo Ordóñez et al., "Virtual community of practice to potentiate knowledge and skills for building mobile applications in computer science students", 2016 8th Euro American Conference on Telematics and Information Systems (EATIS), de Yuli Andrea Rodríguez. Cartagena, Colombia: IEEE, 2016.

40 Cristian Ordóñez et al., "Strengthening Competencies for Building Software, Through a Community of Practice", en: Advances in Computing. CCC 2017. Communications in Computer and Information Science, eds. Andrés Solano y Hugo Ordóñez (Basilea: Springer, 2017). 415-426. 
Por esto, cobra fuerza la necesidad de propiciar un espacio que les permita desarrollar competencias a los estudiantes del programa profesional de Ingeniería de Sistemas en la construcción de software a través de equipos, como una oportunidad de innovar no únicamente en la educación, sino en otras áreas de conocimiento.

\section{Metodología}

La investigación que soporta este artículo se realizó con el paradigma cuantitativo, con un enfoque empírico-analítico de tipo descriptivo y propositivo. La población objeto de estudio corresponde a dieciséis (16) estudiantes de séptimo y veinticuatro (24) estudiantes de octavo semestre del programa de Ingeniería de Sistemas, de la Universidad Mariana. Las técnicas que se utilizaron para la recolección de la información fueron: encuesta y taller. Para el análisis de la información, se utilizó la estadística descriptiva como técnica. Las variables analizadas fueron: fase, rol, artefacto, lineamiento y herramienta.

El trabajo se desarrolló en tres etapas: en la primera, se logró identificar el nivel de desempeño que tienen los estudiantes de Ingeniería de Sistemas en la construcción de software basada en equipos; en la segunda etapa, se conforma una comunidad virtual de práctica para el desarrollo de competencias en construcción de software basada en equipos; por último, se determina el nivel de aporte de la comunidad virtual de práctica, en el desarrollo de competencias en la construcción de software basada en equipos, a los miembros de la comunidad.

En una primera etapa, se estableció el nivel de desempeño que tienen los estudiantes antes de formar parte de la comunidad virtual de práctica (pre-test). Posteriormente, se pone en funcionamiento la comunidad y, después de seis meses de funcionamiento, se vuelve a identificar el nivel de desempeño alcanzado para construir software en equipo desde un enfoque ágil (post-test).

Los instrumentos se construyen y validan a través de las fases: identificar las competencias por cada variable, establecer los niveles de desempeño de cada competencia, operativizar el desempeño mediante preguntas, elaborar el cuestionario, validar el cuestionario a través de juicio de expertos, realizar ajustes al cuestionario de acuerdo con las sugerencias de los expertos, desa- 
rrollar una prueba piloto, y efectuar ajustes al cuestionario en relación con las observaciones planteadas en la prueba piloto. La participación de las personas en esta investigación se sujetó a la aprobación y firma de un documento, donde se informa el lugar y objetivo del estudio, la elegibilidad, el procedimiento, los riesgos, los beneficios, las compensaciones, la libertad de remoción y el consentimiento voluntario.

\section{Resultados}

A continuación, se presentan los resultados obtenidos presentando la estructura y organización de la comunidad virtual de práctica y una comparación entre los niveles de logro obtenidos por los integrantes antes y después de participar como miembros activos de la comunidad.

\subsection{Comunidad virtual de práctica propuesta}

En esta sección, se describe cómo se conformó una comunidad virtual de práctica para el desarrollo de competencias en construcción de software basada en equipos, en la Universidad Mariana. Según Amin y Roberts ${ }^{41}$ y Ordóñez et al. ${ }^{42}$ una comunidad de práctica se estructura a través de tres elementos: comunidad, práctica e identidad.

La estructura de la comunidad virtual de práctica propuesta se presenta en la Figura 1 y muestra los elementos que la componen y las relaciones entre los elementos.

\footnotetext{
$41 \quad$ Ash Amin y Joanne Roberts, "Knowing in action: Beyond communities of practice", Research Policy, vol. 37. No. 2 (2008): 353-369.

42 Cristian Ordóñez et al., "Strengthening Competencies for Building...", 3.
} 
Figura 1. Arquitectura de la comunidad virtual de práctica.

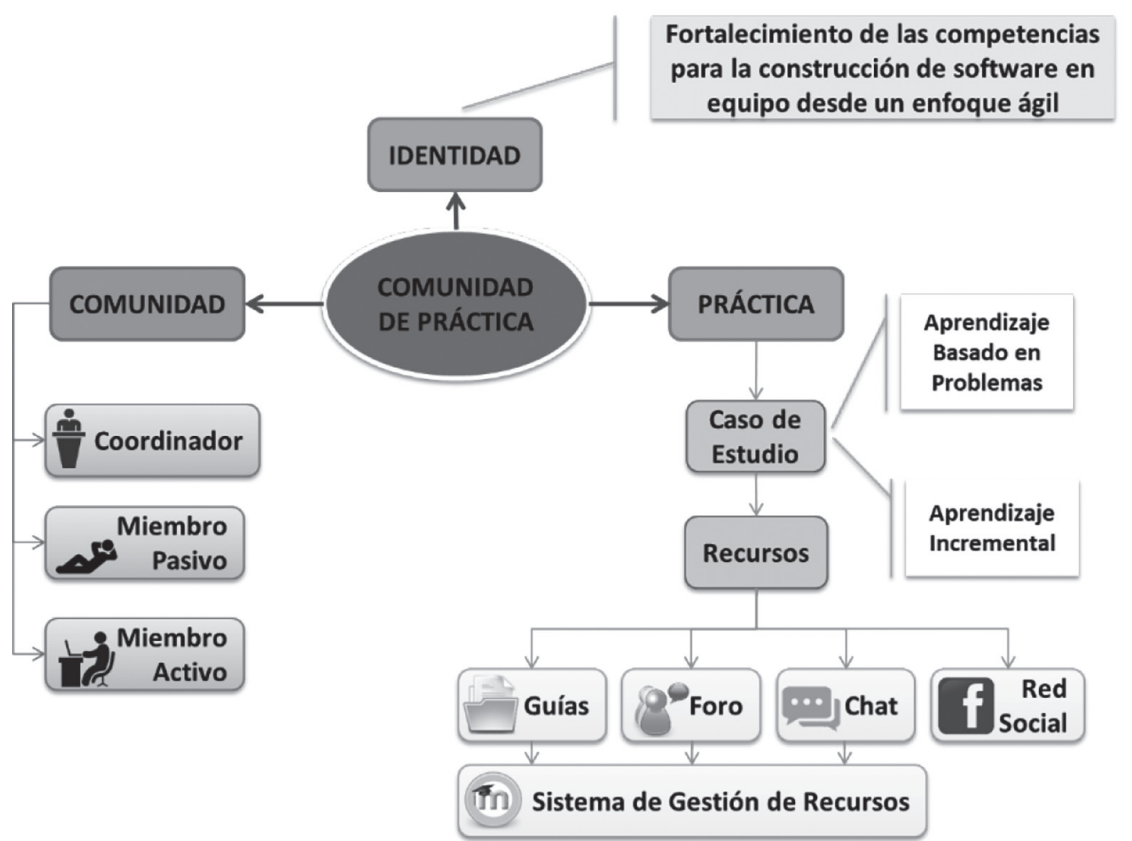

Fuente: esta investigación

A continuación, se describen los elementos y función de cada parte de la estructura de la comunidad.

\subsubsection{Identidad}

La identidad se entiende como el campo de interés compartido por la comunidad; esta identidad crea una motivación para que los estudiantes se reúnan y guíen el aprendizaje de manera autónoma; en este sentido, el campo de interés dentro de la comunidad es potenciar las competencias para la construcción de software en equipo.

El proceso de conformación inicia en la Universidad Mariana, donde se tiene la carrera de Ingeniería de Sistemas, en la cual se realizó una charla motivacional, para dar una explicación a los estudiantes de lo que significa una comunidad virtual de práctica y el propósito que tiene. Posteriormente, se invitó a participar de la experiencia a los estudiantes de séptimo y octavo semestre del programa de Ingeniería de Sistemas, durante el semestre B de 2017 y semestre A de 2018. 


\subsubsection{Práctica}

El elemento práctica es aquel en que los integrantes de la comunidad comparten y dan a conocer su experticia en un tema, además de desarrollar recursos para compartir. ${ }^{43}$ Para guiar la práctica, se adoptó como estrategia didáctica el aprendizaje basado en problemas, donde se plantea fortalecer las competencias para la construcción de software en equipo desde un enfoque ágil, por medio de la solución de problemas reales y significativos. La solución de este tipo de problemas permite que los integrantes de la comunidad desarrollaran las habilidades necesarias para recolectar y analizar diferentes fuentes de información, además de analizar las situaciones reales desde una perspectiva teórica, proponer y evaluar soluciones con utilización de los recursos que tienen disponibles, planificar y proyectar.

Se plantearon tres problemas reales, a través de casos de estudio. El primer caso de estudio corresponde al rediseño de un proceso que es manual, para lo cual deben elaborar un flujograma de trabajo, donde cada integrante debe realizar una actividad que permita al equipo lograr un objetivo. El segundo caso de estudio corresponde a las dificultades que se han presentado en la trazabilidad de las actividades que se desarrollan a partir de la ejecución de un proyecto de tecnología en una organización. El tercer caso de estudio plantea las dificultades que se presentan, en el sistema de información actual, para el préstamo y recuperación de material bibliográfico de una Universidad.

Un aspecto importante para propiciar el desarrollo de las competencias en la comunidad fue los recursos. El recurso principal, sobre el que se basa la estrategia adoptada y a partir del cual se establecieron los demás recursos, fue el caso de estudio, que representa diversas situaciones y problemáticas de la vida real, que pueden estudiar y analizar los estudiantes, con el fin que los miembros de la comunidad generen ideas de solución. Esto exige a los participantes de la comunidad desarrollar habilidades creativas, la capacidad que tienen para innovar y representa un recurso para conectar la teoría con la práctica.

Por otra parte, se lograron identificar y seleccionar casos de estudio que posibilitasen el fortalecimiento de las competencias en los integrantes de la co-

$\overline{43}$ Amin y Roberts, "Knowing in action...", 353-369. 
munidad de manera incremental ${ }^{44}$ y cada caso de estudio corresponde a un nivel de competencia que se debe alcanzar, donde se refuerzan conceptos vistos en un nivel anterior y aplican las habilidades generadas hasta el momento en la solución del problema planteado. Para la comunidad virtual de práctica se crearon y utilizaron tres casos de estudio, como se señaló anteriormente.

Al tener en cuenta los casos de estudio, se elaboraron guías de trabajo, que los integrantes desarrollaron, las que se enfocan en fortalecer los conocimientos y aptitudes para la construcción de software basada en equipo desde un enfoque ágil. Además, se creó un foro como medio para compartir y despejar dudas e inquietudes, que se presentaron en la realización de las guías de trabajo. Así mismo, se puso a disposición un chat para que los integrantes de la comunidad pudieran compartir conocimiento y comunicarse. Otro elemento importante es el referido a las redes sociales, que se utilizaron como estrategia de interacción entre los miembros de la comunidad. Esos recursos, nombrados antes, se soportan en un sistema de gestión de cursos, para acceder a los servicios que la comunidad ofrece.

\subsubsection{Comunidad}

Este elemento se refiere a los miembros que forman parte de ella, los cuales intervienen y comparten un interés común en el tema de trabajo de la comunidad. Los participantes se incluyen en actividades en las cuales comparten conocimientos y se apoyan mutuamente. Según Ordóñez et al.,${ }^{45}$ en una comunidad se presentan estos roles entre los miembros: 1) el coordinador, responsable de organizar eventos y conectar a los miembros de la comunidad; 2) los miembros activos, aquellos que asisten a las reuniones con regularidad y participan en los foros o actividades planteadas en la comunidad; este grupo lo conformaron 40 estudiantes: 16 de séptimo y 24 de octavo semestre del programa de Ingeniería de Sistemas, durante el segundo semestre de 2017 y el primer semestre de 2018; 3) los miembros periféricos, quienes asisten en escasas ocasiones; además, se encargan de observar las interacciones de los miembros activos.

\footnotetext{
44 Jorge Villalobos y Rubby Casallas, Fundamentos de programación. Aprendizaje activo basado en casos (Bogotá: Universidad de los Andes, 2016), https://www.gitbook.com/book/ universidad-de-los-andes/funda mentos-de-programacion/details

45 Cristian Ordóñez et al., "Strengthening Competencies for Building...", 3.
} 


\subsection{Nivel de aporte de la comunidad}

En el cumplimiento de la primera etapa, se identificó el nivel de desempeño de la población antes de la comunidad virtual de práctica (pre-test). Posteriormente, se pone en funcionamiento la comunidad y, después de seis meses de funcionamiento, se vuelve a determinar el nivel de desempeño alcanzado (post-test).

$\mathrm{Al}$ tener en cuenta los elementos que debe poseer un método para propiciar el trabajo en equipo, ${ }^{46}$ las variables analizadas fueron: etapa, que reúne un conjunto de actividades que se realizan para alcanzar un fin; rol, como la función o papel que una persona desempeña en un equipo; artefacto o entregable, como un producto que se realiza y agrega valor a la construcción de software; lineamiento, que se refiere a una tendencia, una dirección o un rasgo que posibilita la toma de decisiones; y herramienta, que corresponde a un elemento que permite soportar y facilitar los procesos de desarrollo de software.

\subsubsection{Contraste competencia cognitiva}

En relación con la dimensión del conocimiento o saber, como se puede observar en la Gráfica 1, se obtuvo un avance en el nivel de desempeño de los estudiantes después de haber interactuado en la comunidad virtual de práctica. En la variable etapa, se logra avanzar de un nivel de desempeño del $90 \%$ al $100 \%$, lo que muestra un avance o incremento del 10\%; es decir, reconocen las etapas y actividades para gestionar un proyecto de construcción de software en equipo, desde el inicio y paso por la ejecución y cierre. En la variable rol, se logra un aumento del 17\%, con identificación de los roles que deben desempeñar los integrantes de un equipo ágil, como son Product owner, Scrum master y Development team. Para la variable artefacto, se logra un incremento del 8\%, al reconocer nuevos productos en la gestión del trabajo en equipo, como: product backlog, Sprint backlog y Done. En la variable herramienta, se identifican nuevas herramientas para brindar soporte a todo el proceso de gestión del trabajo en equipo, a través de Kunagi, con obtención de un incremento en el nivel de desempeño del $23 \%$.

${ }_{46}$ Hernández et al., "Metodología adaptativa basada en Scrum...., 214. 
Es importante tener en cuenta que, en la variable lineamiento, se tuvo una disminución del desempeño en un 4\%; específicamente, en la gestión del trabajo en equipo pierde importancia el uso de indicadores para generar información en la etapa de cierre, que posibilite tomar decisiones de manera informada, por lo cual se sugiere utilizar otras estrategias de intervención.

Gráfica 1. Contraste en los conocimientos antes y después de la comunidad virtual de práctica.

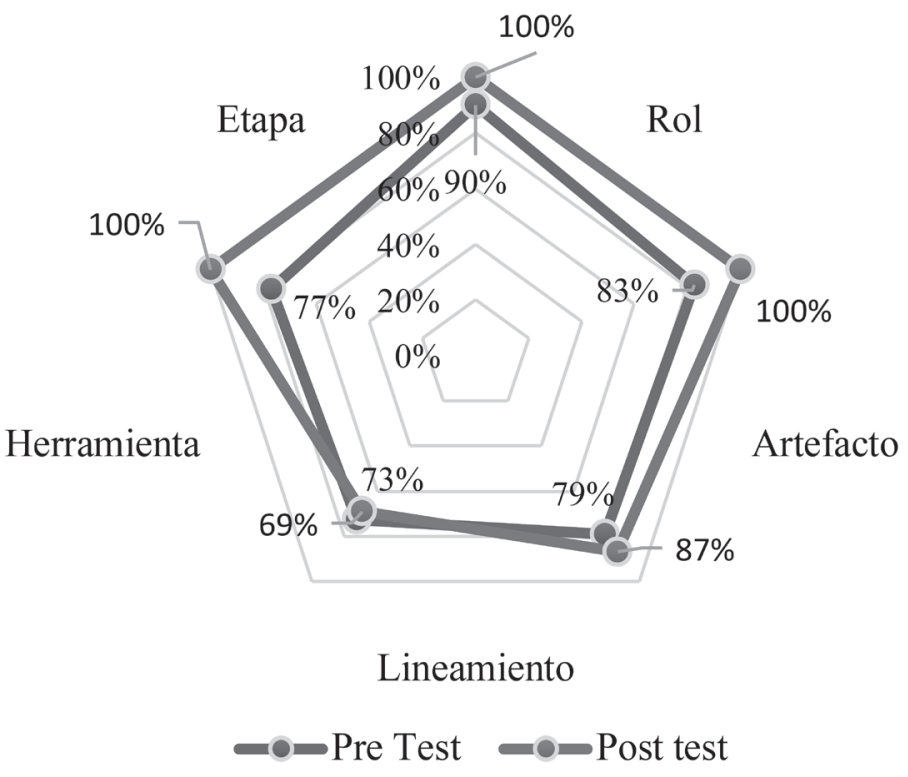

Fuente: esta investigación

\subsubsection{Contraste competencia aptitudinal}

En relación con el saber-hacer, se tuvo un mejoramiento del desempeño en 4 de las 5 variables evaluadas, lo cual permite afirmar que los miembros de la comunidad virtual de práctica lograron consolidar un nivel de coherencia entre el nivel de conocimientos y su aplicación.

Como se puede observar en la Gráfica 2, se puede determinar que, antes de participar de la comunidad de práctica, existía una gran dificultad en transferir los conocimientos a la práctica; es decir, una dificultad en utilizar los conocimientos para solucionar problemas reales. 
Para la variable etapa, se logra que los estudiantes dejen de centrar el trabajo en la fase de ejecución únicamente y se pasase a planear y organizar el trabajo en equipo en la etapa de inicio; y una vez finalizara la ejecución, se evalúe el trabajo realizado y la forma como se logra, a través de las actividades Sprint review y Retrospective. En cuanto a la variable artefacto, los estudiantes, al abordar las tres fases del proceso de elaborar software en equipo desde un enfoque ágil, utilizan artefactos para cada fase, acción que les permite avanzar de un nivel de logro alcanzado del $33 \%$ al $83 \%$. Por ejemplo, en la etapa de inicio, para la planeación, se elabora el product backlog, se prioriza el trabajo y se determina la complejidad. En la etapa de ejecución, se define el Done, con determinación de los artefactos que construyen y agregan valor a la elaboración de un producto software. Finalmente, en la etapa de cierre, se elabora un documento, donde se analiza la relación entre el trabajo planeado y el logrado; se realiza una nueva priorización para una nueva iteración e incremento. En la retrospectiva, los estudiantes identifican las acciones que han venido realizando y deben seguir haciendo, las acciones que están haciendo y no deben continuar; y las acciones que no están haciendo y deben hacer.

Para la variable lineamiento, se desarrollan las actividades Sprint review y retrospective como parte de la etapa de cierre, acciones que generan información para planear un nuevo incremento e iteración. No obstante, se identifica, en la ejecución, que sigue sin ser de interés de los estudiantes recopilar información a través de indicadores.

Por último, para la variable herramienta, en el pre-test, los estudiantes no utilizan ninguna herramienta para gestionar la construcción de software a través de equipos. En el post-test, los estudiantes utilizan la herramienta Kunagi para soportar las tres etapas (inicio, ejecución y cierre). 
Gráfica 2. Contraste en las aptitudes antes y después de la comunidad virtual de práctica.

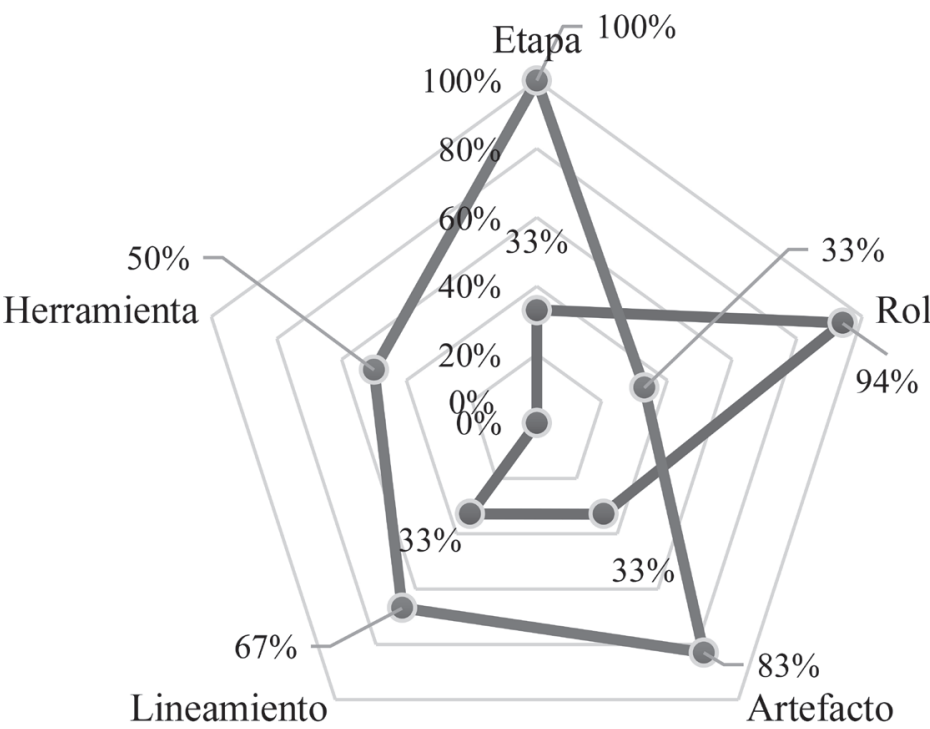

-0 Pre Test $=0$ Post test

Fuente: Esta investigación

En cuanto a la variable rol, los estudiantes, en el pre-test, recurren a la determinación de roles para gestionar la construcción de software en equipo; sin embargo, a pesar de reconocer los roles que se plantean desde el método ágil Scrum, no se ponen en práctica para solucionar un problema real de manera incremental, acción que demuestra un decremento del $61 \%$ en el nivel de desempeño alcanzado por los estudiantes en el pre-test.

\section{Conclusiones}

Se conforma una comunidad virtual de práctica estructurada mediante una identidad, una práctica y se establecieron los roles que deben cumplir los integrantes, para poder transformar de manera positiva el nivel de conocimientos y habilidades de los miembros que la conforman. En este caso, la identidad po- 
sibilitó fortalecer el trabajo tradicional realizado en el programa de Ingeniería de Sistemas, en relación con el desarrollo de competencias para la construcción de software en equipo desde un enfoque ágil.

La comunidad virtual de práctica permite avanzar en los niveles de logro alcanzados para la dimensión cognitiva, en las competencias requeridas para construir software desde un enfoque ágil en los integrantes que se desempeñaron como miembros activos en la comunidad, con excepción de la variable lineamiento, donde el desempeño permaneció estático.

El enfoque para el desarrollo de competencias basado en problemas y los recursos construidos para que los utilizaran los miembros activos de la comunidad virtual de práctica, permitieron avanzar en los niveles de logro alcanzados para la dimensión aptitudinal, en las competencias requeridas para construir software desde un enfoque ágil, con excepción de la variable rol, donde se promueve obviar la importancia de esos roles.

La comunidad virtual de práctica permitió avanzar en los niveles de logro alcanzados para la dimensión cognoscitiva, con excepción de la variable lineamiento; por esta razón, se recomienda definir nuevos casos de estudio donde el trabajo de los miembros de la comunidad se centrase en recopilar datos en la etapa ejecución, analizarlos en la etapa de cierre y generar información que les permita tomar decisiones.

La comunidad virtual de práctica se conformó con el fin de propiciar el desarrollo de competencias para elaborar software a través del trabajo en equipo; debido a que los resultados obtenidos en relación con las variables: lineamiento y rol no fueron los esperados, se recomienda proponer nuevas investigaciones, donde se conformen comunidades virtuales de práctica, que se combinen, apoyen o nutran con el trabajo presencial, en busca de fortalecer el uso de roles e información, para avanzar en la efectividad y eficiencia de los equipos. 


\section{Referencias}

Amin, Ash, y Roberts, Joanne. "Knowing in action: Beyond communities of practice”. Research Policy. Vol. 37. No 2, (2008): 353-369.

Bozu, Zoia, y Muñoz, Francesc. "Creando comunidades de práctica y conocimiento en la Universidad: una experiencia de trabajo entre las universidades de lengua catalana". Universities and Knowledge Society Journal. Vol. 6. No. 1, (2009): 1-10.

Brooks, Frederick. The Mythical Man-Month: Essays in Software Engineering. Boston: Addison-Wesley, 1995.

Cockburn, Alistair. Effectiveness of different modes of communication graph. 2015. http://alistair.cockburn.us/Effectiveness + of + different + modes + of +communication+graph.gif

Dascalu, Maria-Iuliana et al. "Improving e-learning communities through optimal composition of multidisciplinary learning groups". Computers in $\mathrm{Hu}$ man Behavior. No. 30, (2014); 362-371.

DeMarco, Tom. Slack: Getting past burnout busywork and the myth of total efficiency. New York: Broadway Books, 2001.

Dumitru, Daniela, y Enăchescu, Vladimir. "Communities of Practice as a Mean for Decentralization". Procedia - Social and Behavioral Sciences. No. 187 (2015): 752-756.

Fedesoft. "Visión estratégica del sector de software y servicios asociados plan de mercadeo y ventas regionalizado del sector en Colombia". Bogotá: Mintic y Colciencias, 2013.

Forero, Aracely, y Mesa, Fredy. "Reflexiones para la historia y prospectiva de la virtualidad en la educación superior colombiana". Historia de la Educación Colombiana. Vol. 15. No. 15, (2012): 215-236. 
Hernández, Giovanni et al. "Metodología adaptativa basada en Scrum: Caso empresas de la Industria de Software en San Juan de Pasto - Colombia". Revista Tecnológica-ESPOL. Vol. 28. No. 5, (2015): 211-223.

Hernández, Giovanni et al. «Scrum y Peopleware: elementos clave para la gestión en la construcción de software.» Risti. No. E19, (2019): 265-277.

Infosys y Universidad EAFIT. Brecha de Talento Digital Infosys - Universidad EAFIT. Universidad EAFIT e Infosys Limited, 2014.

Jiménez-Zarco, Ana Isabel et al. "The co-learning process in healthcare professionals: Assessing user satisfaction in virtual communities of practice". Computers in Human Behavior. Vol. 51. Parte B, (2015): 1303-1313.

Kim, Hyung Jin et al. "Third-party mobile app developers' continued participation in platform-centric ecosystems: An empirical investigation of two different mechanisms". International Journal of Information Management. Vol. 36. No. 1, (2016): 44-59.

Kummitha, Rama y Majumdar, Satyajit. "Dynamic curriculum development on social entrepreneurship - A case study of TISS". The International Journal of Management Education. Vol. 13. No. 3, (2015): 260-267.

Lupu, Daciana, y Laurenţiu, Andreea. "Using New Communication and Information Technologies in Preschool Education". Procedia - Social and Behavioral Sciences. No. 187, (2015): 206-210.

Narayanan, S, V Jayaraman, Y Luo, y J Swaminathan. "The antecedents of process integration in business process outsourcing and its effect on firm performance". Journal of Operations Management. Vol. 29. No. 1, (2011): 3-16.

Ordóñez, Camilo et al. "Virtual community of practice to potentiate knowledge and skills for building mobile applications in computer science students". En 2016 8th Euro American Conference on Telematics and Information Systems (EATIS), de Yuli Andrea Rodríguez. Cartagena, Colombia: IEEE, 2016. 
Ordoñez, Cristian et al. "Strengthening Competencies for Building Software, Through a Community of Practice". En Advances in Computing. CCC 2017. Communications in Computer and Information Science, editado por Solano, Andrés, y Ordóñez, Hugo. Basilea: Springer, 2017. 415-426.

Pan, Yonggang et al. "Integrating social networking support for dyadic knowledge exchange: a study in a virtual community of practice". Information \& Management. Vol. 52. No. 1, (2015): 61-70.

Parnin, Chris, y Rugaber, Spencer. "Resumption strategies for interrupted programming”. Software Quality Journal. Vol. 19. No. 1, (2011): 5-34.

Putnam, Lawrence. "A general empirical solution to the macro software sizing and estimating problem". IEEE transactions on Software Engineering. Vol. SE-4. No. 4, (1978): 345-361.

Ratzinger-Sakel, Nicole V. S., y Gray, Glen L. "Moving toward a learned profession and purposeful integration: Quantifying the gap between the academic and practice communities in auditing and identifying new research opportunities". Journal of Accounting Literature. 35 (2015): 77-103.

Rheingold, Howard. La comunidad virtual: una sociedad sin fronteras. Barcelona: Gedisa, 1996.

Robertson, Christopher J. et al. "An analysis of the predictors of software piracy within Latin America". Journal of Business Research. Vol. 61. No. 6, (2008): 651-656.

Rodríguez, Darío et al. "Information technology within society's evolution". Technology in Society. No. 40, (2015): 64-72.

Rogo, Francesco et al. "Assessing the performance of open innovation practices: A case study of a community of innovation". Technology in Society. No. $38,(2014): 60-80$.

Salinas, Jesús. "Comunidades virtuales y aprendizaje digital”. Edutec'03 (2003): $1-21$. 
Tobón, Sergio. La formación basada en competencias en la educación superior: el enfoque complejo. México: Universidad Autónoma de Guadalajara, 2008 .

Villalobos, Jorge, y Casallas, Rubby. "Fundamentos de programación, aprendizaje activo basado en casos". 2016. https:/www.gitbook.com/book/universidad-de-los-andes/fundamentos-de-programacion/details.

Wenger, Etienne. "Communities of Practice: Learning as a Social System". Systems thinker. Vol. 9. No. 5, (1998): 1-10. 